\title{
Status of Ground-Based Gravitational Wave Detectors
}

\author{
Peter R. Saulson \\ Department of Physics, Syracuse University, Syracuse, NY 13244-1130 USA
}

\begin{abstract}
Gravitational wave detectors on the ground are now operating full-time at unprecedented sensitivity. Detection of gravitational waves by ground-based detectors is expected, if not from this generation, then from its successors that will start construction within a few years.
\end{abstract}

Keywords: gravitational wave detection, interferometer, resonant-mass detector

PACS: 95.55.Ym, 04.80.Nn

\section{INTRODUCTION}

The development of gravitational wave detectors has been a long process, dating to the work of Joseph Weber in the 1960s. [1] Finally, in 2006, ground-based gravitational wave detectors are operating with sensitivities at which it would no longer be a surprise to detect an astrophysical signal from known sources (although it would be considered a bit of luck.) Equally exciting is the fact that a next generation of detectors is about to be constructed that are expected to see many signals, perhaps at a rate of more than one per day. That generation of detectors will come on line in the middle of the next decade, just in time to complement LISA's expected observations from many other sources of gravitational waves.

\section{RESONANT-MASS DETECTORS}

Resonant-mass detectors are Weber's original style of gravitational wave detectors. Over the ensuing decades, sensitivity has been improved by several orders of magnitude. Cryogenic technology has reduced mechanical thermal noise, and also allowed the use of ultra-quiet SQUID amplifiers. New vibration isolators and transducers have also played an important role in the achievement of rms strain sensitivities of about $10^{-19}$.

The AURIGA detector, shown in Figure 1, is one of this latest generation of resonant-mass detectors. It is located in Legnaro. [2] The Rome group operates two comparable detectors, NAUTILUS in Frascati and EXPLORER at CERN. [3] The Louisiana State group operates ALLEGRO on the LSU campus in Baton Rouge. [4] 
Resonant-mass detectors have their sensitivity concentrated in a band around the bar's resonant frequency, or in a pair of such bands around the coupled resonances of the bar-transducer system. The current generation exhibits sensitivity of about $10^{-21} / \mathrm{rtHz}$, in bands measuring tens of hertz wide.

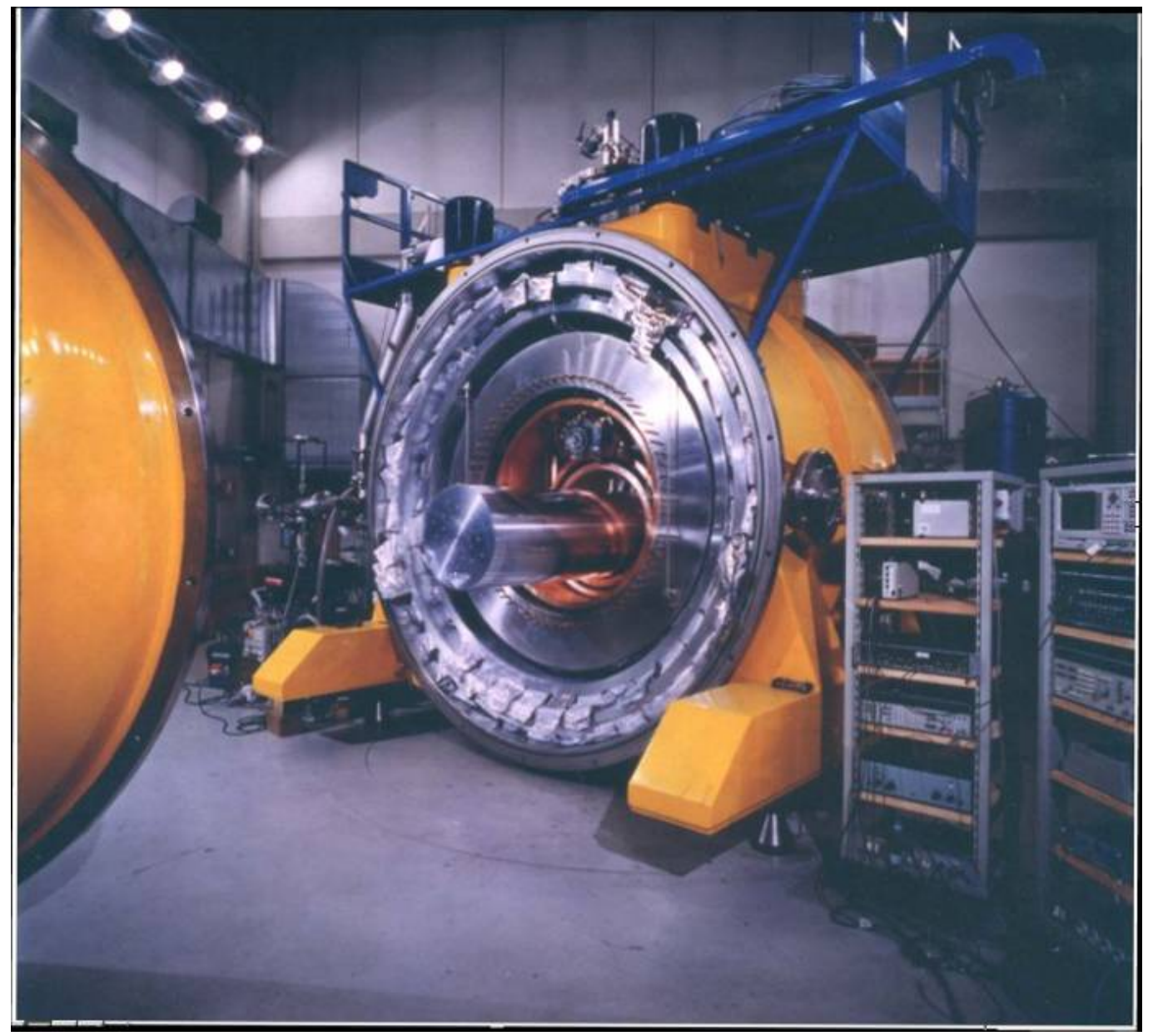

FIGURE 1. The resonant-mass detector AURIGA, with the resonant bar itself seen installed in the cryostat before being closed up and cooled down.

The technology of resonant-mass detectors is subtle and demanding, but in its present state of development is remarkably robust and reliable. This is reflected in an excellent duty factor for observation, limited mainly by the need to periodically refill the cryostats.

Another outstanding feature of the performance of this generation of resonant-mass detectors is the good statistical character of the output. A Gaussian model accounts for the bulk of the events, with only a small tail of non-Gaussian events at large amplitude.

In recent years, all of the resonant-mass detector groups have analyzed their data together as one global network, the International Gravitational Event Collaboration. 
The most recent paper from IGEC set an upper limit on the arrival rate of gravitational wave bursts that is still unsurpassed in the rate limit set on relatively strong bursts. [5]

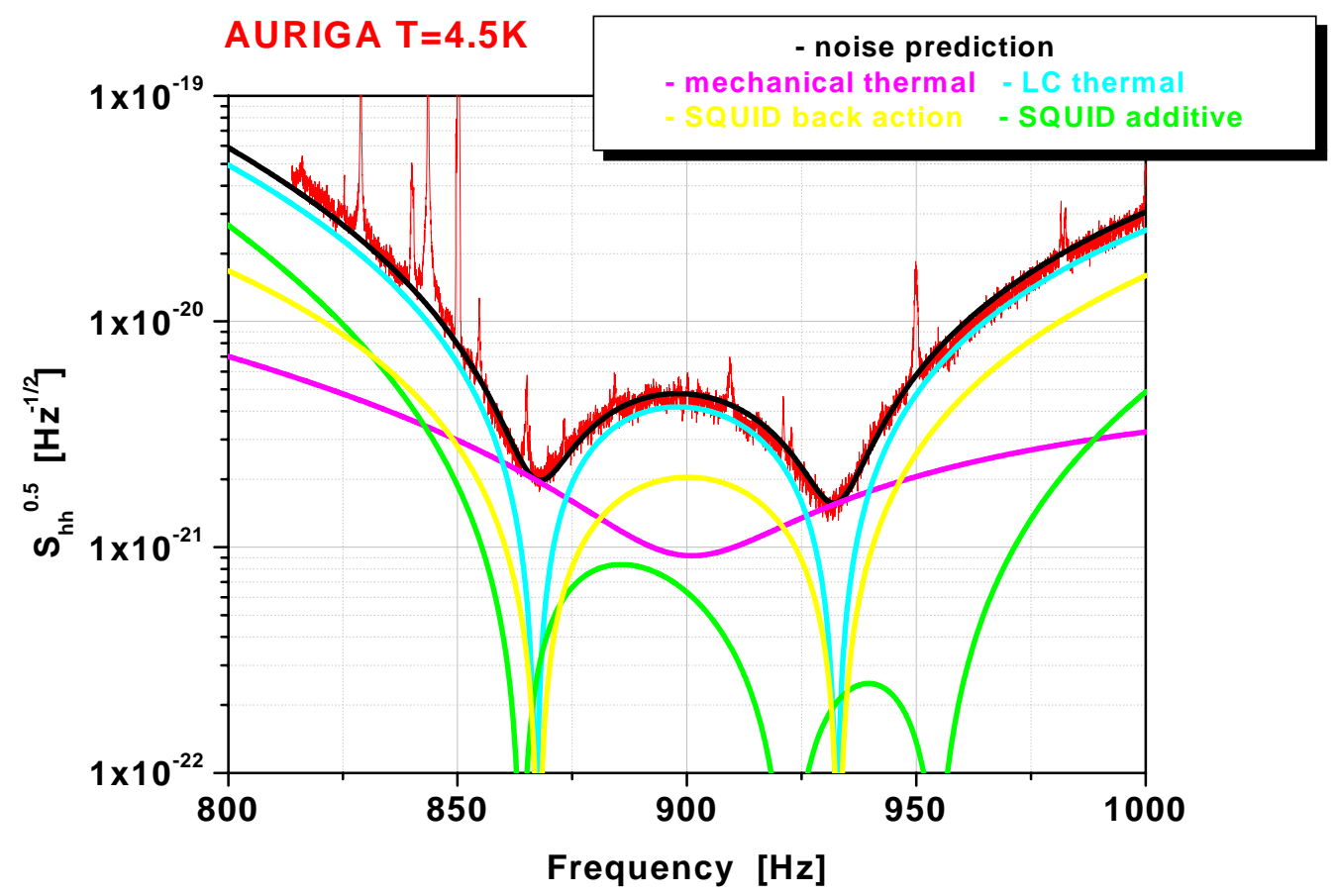

FIGURE 2. The noise spectrum of the AURIGA resonant-mass detector. Note how well the noise is modeled by known effects.

\section{INTERFEROMETERS}

The newer and more sensitive style of ground-based gravitational wave detectors are the interferometers. They, too, have a long history; the pioneers Rainer Weiss [6] and Robert Forward [7] were motivated by Weber's work to aim for detectors that would be both more sensitive and also sensitive over a wider band of frequencies. In the present decade, that hope has finally come to fruition. Several interferometers of kilometer scale are now in operation or about to come on line.

The key advantage of interferometers is their ability to use test masses whose separation can be much farther apart than the ends of a bar (whose length is limited by the need to match the resonant frequency to the expected frequency of a gravitational wave.) Interferometric sensing enables comparison of the lengths of perpendicular arms over distances limited only by practical considerations. Resonant optical cavities allow light to spend time in the arms comparable to the period of the wave, and additional optical cavities return light for multiple passes through the arms (power recycling) and return signal sidebands for enhancement (signal recycling.) Various combinations of these techniques are applied in the interferometers operating today; they lead to sensing-noise-limited sensitivities of $10^{-22} / \mathrm{rtHz}$ or less, from frequencies in the 10s of hertz up to $1 \mathrm{kHz}$ or higher. Low frequency sensitivity is limited by 
mechanical displacement noise of the individual test masses, chiefly seismic noise and mechanical thermal noise.

It should be obvious how these detectors foreshadow many of the key features of LISA; it is also clear how their limitations point to some of the key advantages of moving to the space environment.

\section{LIGO}

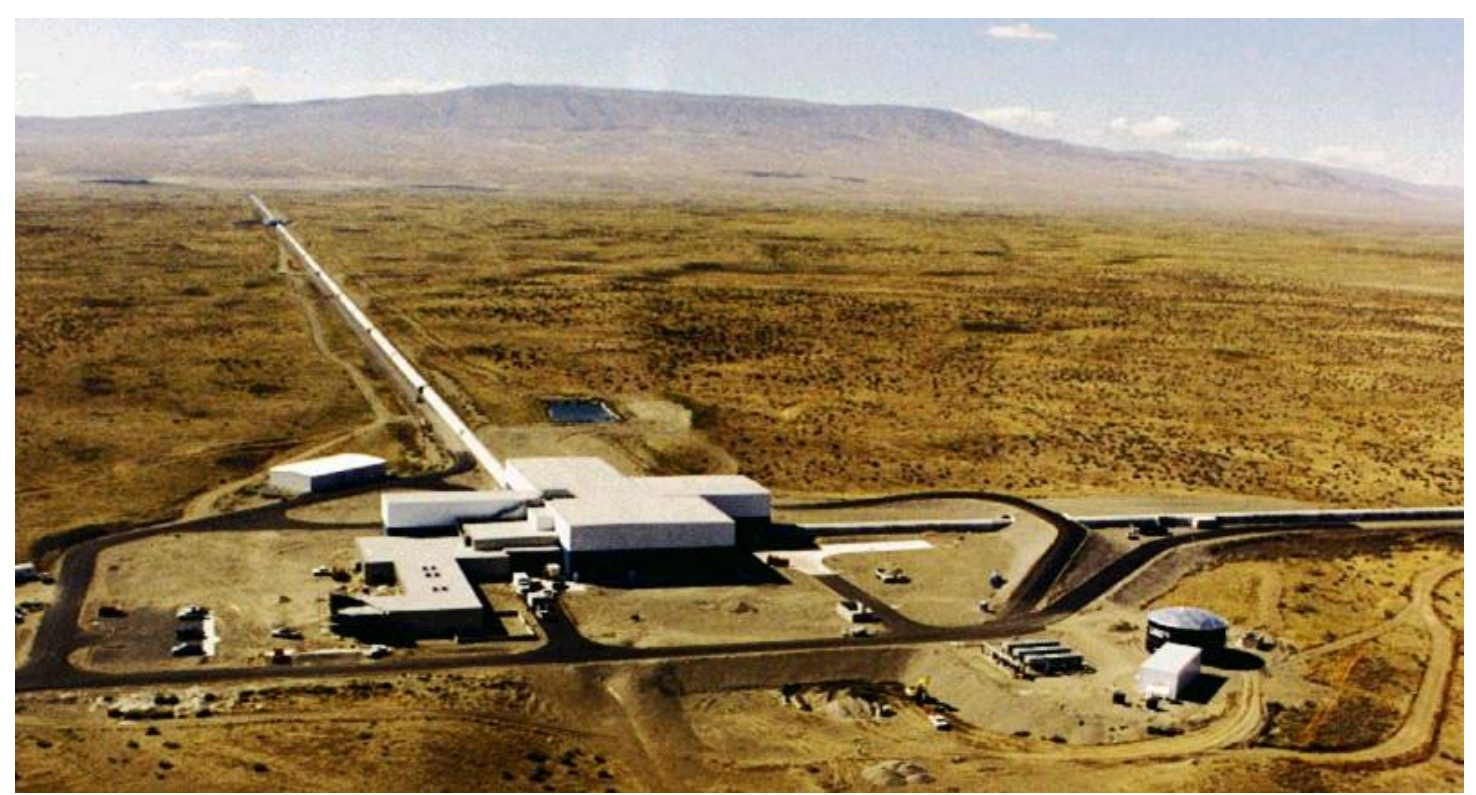

FIGURE 3. LIGO Hanford Observatory, seen from the air. One of its two $4 \mathrm{~km}$ arms can be seen stretching toward the horizon on the left hand side of the figure.

At the time of this writing, the best performance among ground-based detectors has been achieved by the interferometers of the Laser Interferometer Gravitational Wave Observatory (LIGO) in the United States. LIGO has two sites, one in Hanford WA and the other in Livingston LA. LIGO Livingston Observatory has a $4 \mathrm{~km}$ Michelson interferometer, while LIGO Hanford Observatory has both a $4 \mathrm{~km}$ interferometer and a $2 \mathrm{~km}$ interferometer sharing the same beam tube. All three interferometers use FabryPerot cavities in the arms to optimize light storage time, and also use power recycling to increase the effective laser power by about a factor of 50. [8]

Since 2002, the LIGO interferometers have been used in a series of data-collection runs called Science Runs S1 through S5. Performance of the interferometers during each of those runs is shown in Figure 4. The latest run, S5, is going on now, and is intended to collect a year's worth of coincident data from the two LIGO sites. The run began in November 2005, after LIGO had demonstrated achievement of its design goal, a strain spectral density of $10^{-22} / \mathrm{rtHz}$ over a bandwidth of greater than $100 \mathrm{~Hz}$. As Figure 4 demonstrates, the noise is as low as the design goal over almost the entire band from $50 \mathrm{~Hz}$ to several $\mathrm{kHz}$.

The LIGO Scientific Collaboration has produced well over a dozen papers on the results obtained from searches to date. [9] 


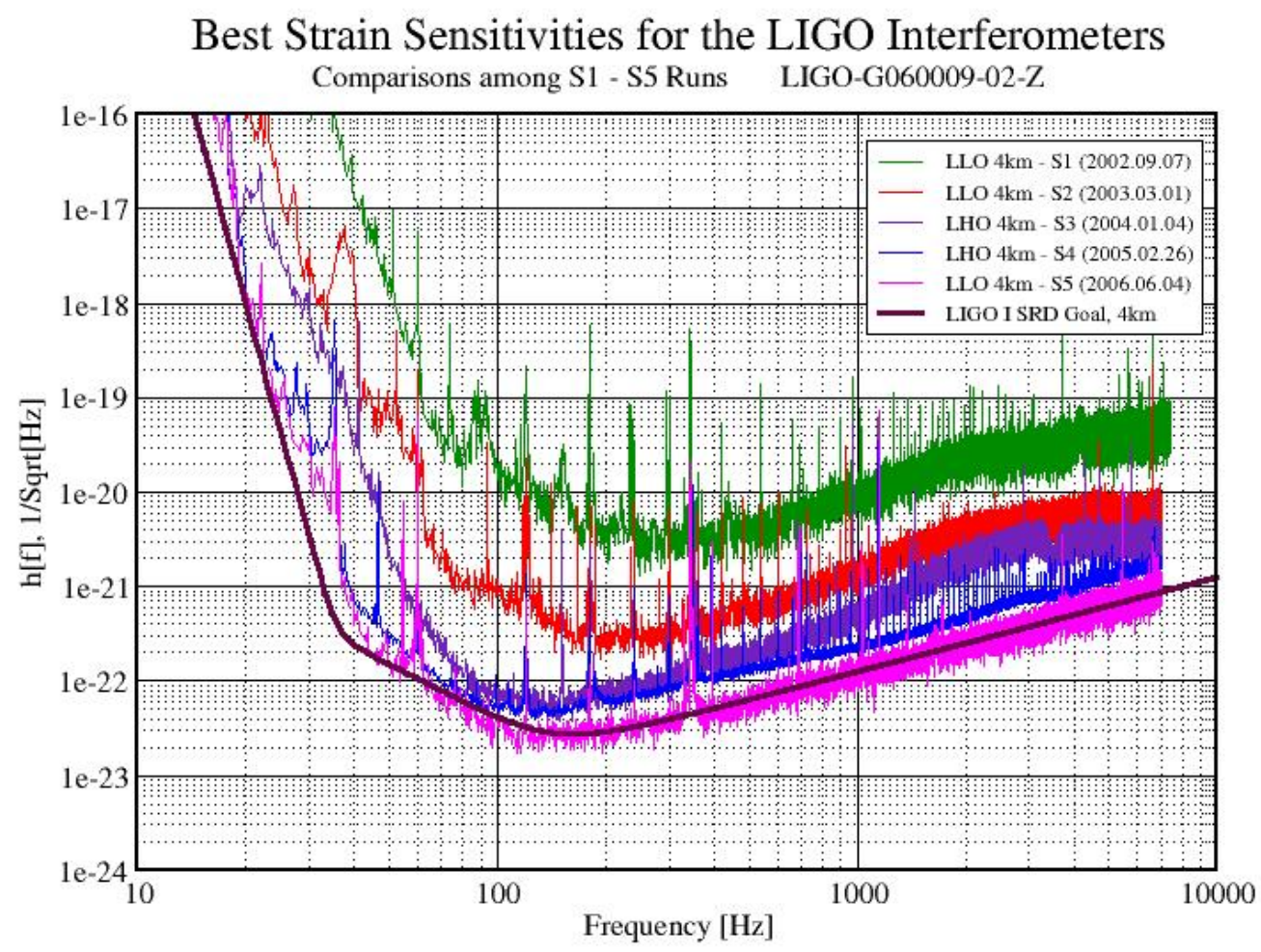

FIGURE 4. Performance of the LIGO interferometers during Science Runs S1 - S5. The original design goal is shown as the bold line.

\section{GEO, TAMA, and Virgo}

The British-German GEO Collaboration has built GEO600, a 0.6 km interferometer located outside of Hannover. It employs simple two-round-trip arms, but uses not only power recycling but also signal recycling to achieve very good performance. So far, noise has been reduced below $10^{-21} / \mathrm{rtHz}$ from $150 \mathrm{~Hz}$ to $2 \mathrm{kHz}$. [10] Starting in May 2006, GEO joined the S5 Science Run on a full-time basis. (GEO belongs to the LIGO Scientific Collaboration.) Late in 2006, another commissioning run is planned in order to come closer to design sensitivity (3 times lower than present noise at high frequencies, with good performance also extending down to about $40 \mathrm{~Hz}$.)

TAMA300 is a $0.3 \mathrm{~km}$ interferometer built by a collaboration of Japanese scientists, located in the outskirts of Tokyo. It employs Fabry-Perot cavities in its arms and also power recycling. It has achieved a broadband sensitivity of about $310^{-21} / \mathrm{rtHz}$ from about $700 \mathrm{~Hz}$ to $3 \mathrm{kHz}$. [11] At the time of this writing, TAMA300 is installing upgraded seismic isolation. The TAMA Collaboration hope to operate the interferometer again soon with improved sensitivity.

Virgo, a $3 \mathrm{~km}$ interferometer built by a French-Italian collaboration near Pisa, is now in advanced stages of commissioning. The Virgo interferometer employs FabryPerot cavities in its arms, as well as power recycling. It is expected to achieve a 
sensitivity comparable to LIGO over a wide band of frequencies, and at low frequencies it has the capability to perform substantially better - Virgo has installed a seismic isolation system (the Super Attenuator) capable of making seismic noise negligible at all frequencies above $3 \mathrm{~Hz}$. [12]

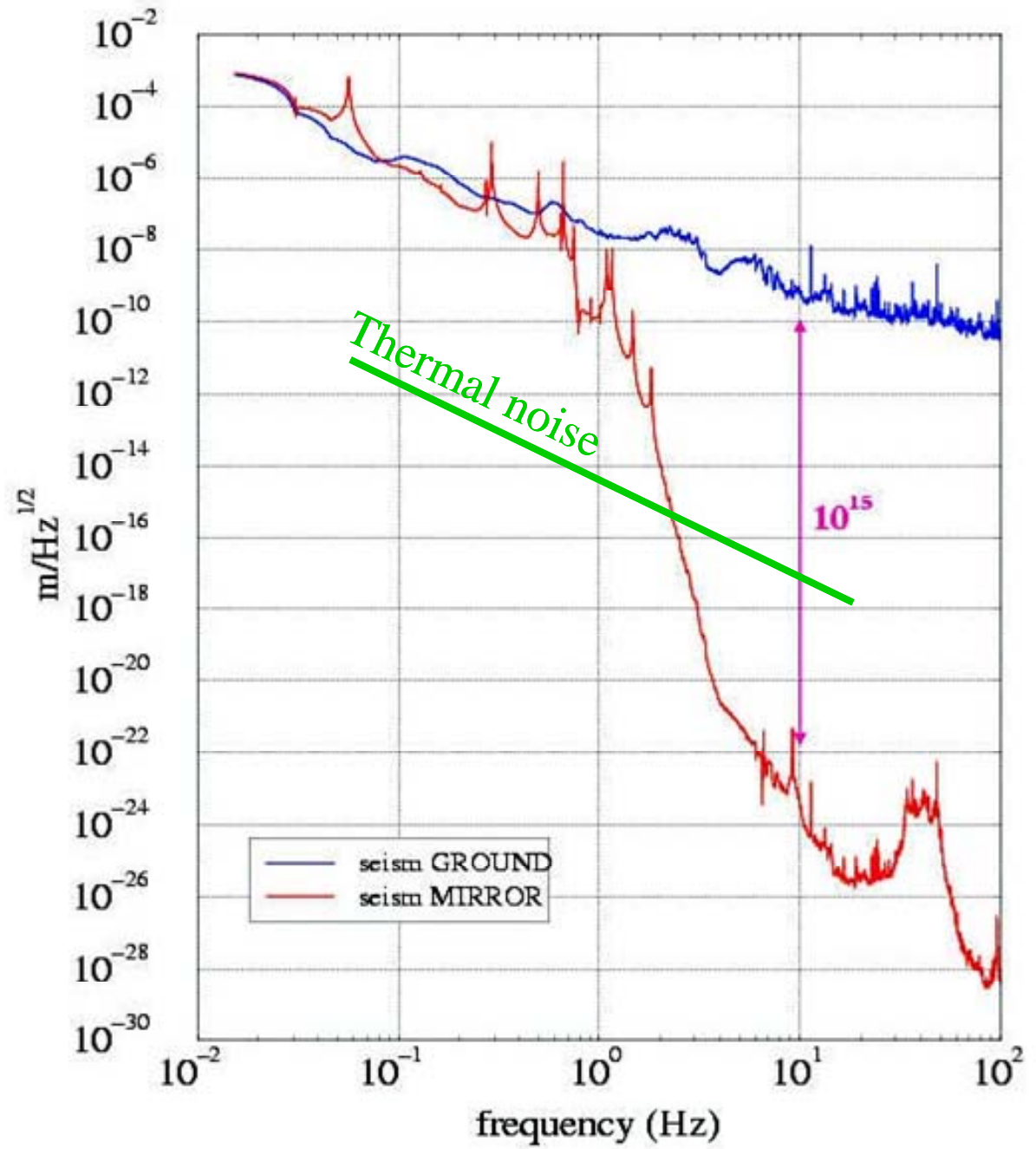

FIGURE 5. Virgo incorporates seismic isolation with very high performance. Seismic noise should be negligible in its noise spectrum down to about $3 \mathrm{~Hz}$.

\section{PROSPECTS FOR THE FUTURE}

Over the next several years, ground-based interferometers are expected to observe as a global network for long periods of time. The LIGO Scientific Collaboration and the Virgo Collaboration are hoping to soon conclude an agreement that would have them carry out all of their data analysis together; this would bring together the data from the three LIGO interferometers and from GEO600 with that from Virgo, and would meld the talent from all of those teams. Such a network would allow the 
determination of the position from which a signal came, and also permit the reconstruction of both polarizations of the waveform.

In the next decade, another generation of interferometers is planned that will have sensitivity that is better by an order of magnitude. Figure 7 illustrates the improvement foreseen from Advanced LIGO. This project has been approved by the National Science Board; we expect to hear in early 2007 that a start for the project has been included in the U.S. budget for FY 2008. (Substantial contributions from the U.K. and from Germany have already been made.) If so, then new hardware would be installed at the LIGO sites starting in early 2011, with new data expected in 2014.

On a similar timescale, Advanced Virgo is also expected to make a similar advance in sensitivity. Japan's proposed LCGT would also have similar performance.

The importance of this next stage of improvements is that it will bring the sensitivity to a level at which detection of signals is expected to be frequent. Coalescences of binary systems of two neutron stars, two black holes, or one of each, ought to be observed regularly by the elements of the ground-based interferometer network in the middle of the next decade.

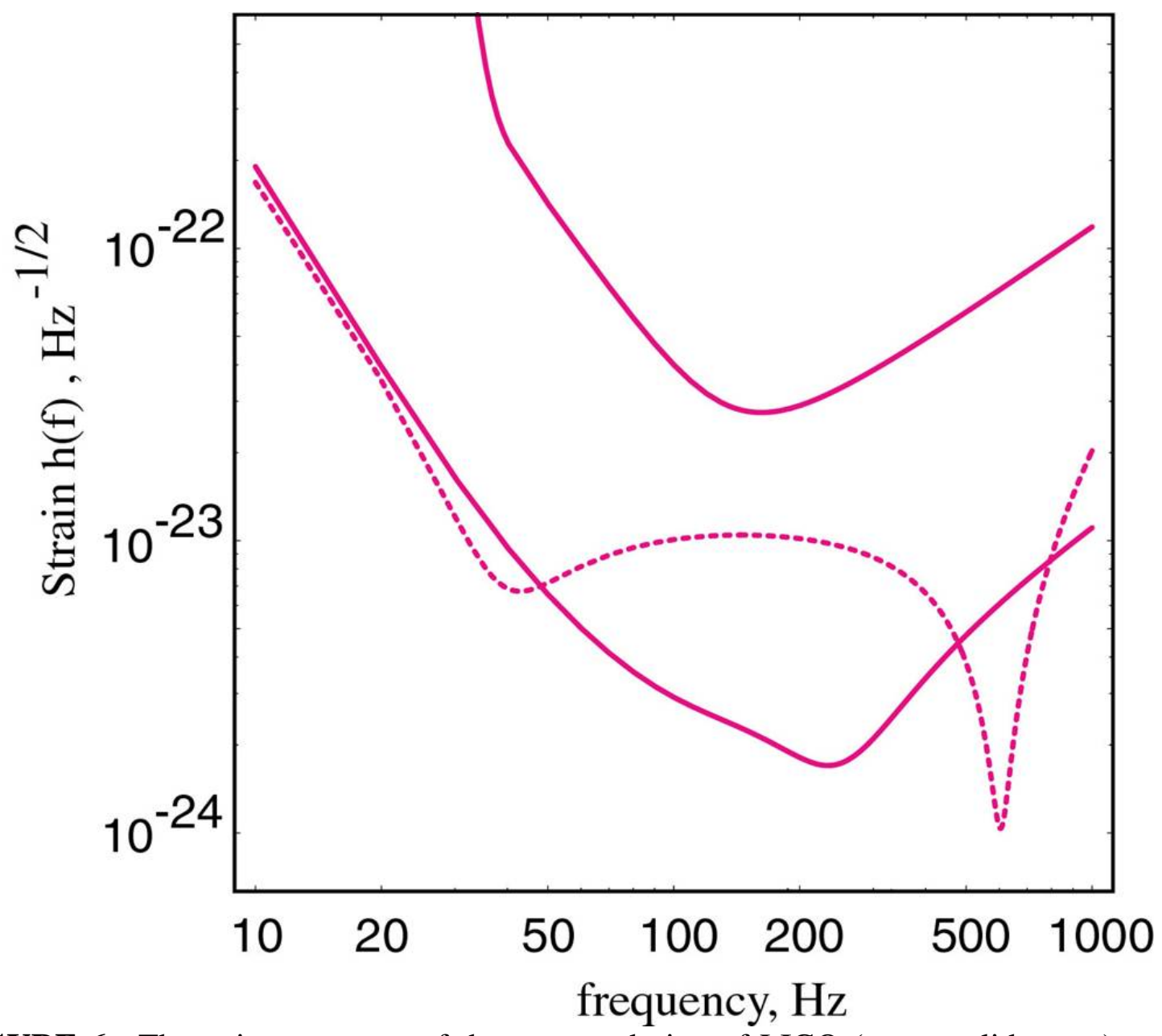

FIGURE 6. The noise spectrum of the present design of LIGO (upper solid curve) compared with two possible noise spectra from different tunings of Advanced LIGO (lower solid curve and dashed curve.) 
Resonant-mass detector technology is not standing still, either. A new concept called DUAL would make measurements of the differential deformations caused by gravitational waves between two nested resonators of different fundamental resonant frequencies. In the band between those resonances, it looks possible to obtain very good broadband sensitivity. [13] Present designs put that sensitive band between 3 and $5 \mathrm{kHz}$, where interferometers' sensitivity is not poorer than near $100 \mathrm{~Hz}$. Thus, the resonant technology could play an important role, for example in studies of neutron star resonant frequencies.

\section{ACKNOWLEDGMENTS}

The author gratefully acknowledges the support of the U.S. National Science foundation both for his own research under grant PHY-0140335, and for its support of LIGO. He also wishes to thank his many colleagues around the globe, and to thank their funding agencies on their behalf.

\section{REFERENCES}

1. J. Weber, Phys. Rev. 117, 306 (1960); J. Weber, Phys. Rev. Lett. 22, 1320 (1969).

2. L. Baggio et al., Phys. Rev. Lett. 94, 241101 (2005).

3. P. Astone et al., Class. Quantum Grav. 23, S57 - S62 (2006).

4. I. S. Heng et al., Class. Quantum Grav. 19, $1889-1895$ (2001).

5. P. Astone et al., Phys. Rev. D 68, 022001 (2003).

6. R. Weiss, Quarterly Progress Report, MIT Research Lab of Electronics 105, 54 (1972).

7. G. E. Moss, L. R. Miller, and R. L. Forward, Appl. Opt. 10, 2495 (1971); R. L. Forward, Phys. Rev. D 17, 379 (1978).

8. B. Abbott et al., Nucl. Instrum. Meth. A517, 154-179 (2004).

9. Links to these papers can be found at http://www.ligo.org/results/.

10.B. Willke et al., Class. Quantum Grav. 21, S417 - S423 (2004).

11. R. Takahashi, Class. Quantum Grav. 21, S403 - S408 (2004).

12. F. Acernese et al., Journal of Physics: Conference Series 39, 32 - 35 (2006).

13. M. Bonaldi et al., Phys. Rev. D 74, 022003 (2006). 